\title{
Separation enhancement in pinched flow fractionation
}

\section{Vig, Asger Laurberg; Kristensen, Anders}

\section{Published in:}

Applied Physics Letters

Link to article, DOI:

$10.1063 / 1.3028652$

Publication date:

2008

Document Version

Publisher's PDF, also known as Version of record

Link back to DTU Orbit

Citation (APA):

Vig, A. L., \& Kristensen, A. (2008). Separation enhancement in pinched flow fractionation. Applied Physics Letters, 93(20), 203507. https://doi.org/10.1063/1.3028652

\section{General rights}

Copyright and moral rights for the publications made accessible in the public portal are retained by the authors and/or other copyright owners and it is a condition of accessing publications that users recognise and abide by the legal requirements associated with these rights.

- Users may download and print one copy of any publication from the public portal for the purpose of private study or research.

- You may not further distribute the material or use it for any profit-making activity or commercial gain

- You may freely distribute the URL identifying the publication in the public portal

If you believe that this document breaches copyright please contact us providing details, and we will remove access to the work immediately and investigate your claim. 


\title{
Separation enhancement in pinched flow fractionation
}

\author{
Asger Laurberg Vig and Anders Kristensen ${ }^{\text {a) }}$ \\ DTU Nanotech, Department of Micro and Nanotechnology, Technical University of Denmark, \\ Building 345 East, Ørsteds Plads, DK-2800 Kongens Lyngby, Denmark
}

(Received 6 October 2008; accepted 24 October 2008; published online 18 November 2008)

\begin{abstract}
A method for enhancing the separation in the microfluidic size separation technique called pinched flow fractionation (PFF) is demonstrated experimentally and analyzed by numerical calculations. The enhancement is caused by a geometrical modification of the original PFF design. Seven different polystyrene bead sizes ranging from 0.25 to $2.5 \mu \mathrm{m}$ in radius were separated in a PFF and in an enhanced PFF device. The separation in the two types of devices were compared and an amplification in the separation of up to $70 \%$ was achieved. Numerical calculations, which include an edge effect, are used to analyze the device. (C) 2008 American Institute of Physics.
\end{abstract}

[DOI: $10.1063 / 1.3028652$ ]

Microfluidic systems and micrototal analysis systems ( $\mu$-TASs) for biological and chemical analysis have proven to be ideal tools for precise handling of small samples, enabling fast analysis in point-of-care and research perspectives. ${ }^{1,2}$ Sorting and separating nanometer to micrometer sized nonbiological and biological particles represents an important step in many chemical and biological processes. A variety of microchip based separation techniques have been demonstrated. ${ }^{3-14}$

Pinched flow fractionation (PFF) is a microchip based size separation technique for nanometer to micrometer sized particles relying on a laminar flow sheet. The method was first demonstrated by Yamada et al., ${ }^{15}$ where 15 and $30 \mu \mathrm{m}$ polystyrene microspheres were separated. The principle of PFF is illustrated in Fig. 1(a). A sample containing particles is introduced from a bead inlet channel into a narrow channel called the pinch segment. The particles are aligned (pinched) against a wall in the pinch segment, regardless of size, using the fluid flow from a buffer inlet channel. As the particles move into a wider channel, the broadening segment, the distance from the center of mass of the particles to the channel wall is amplified and the particles are separated according to size. PFF has been applied for separation of erythrocytes from blood, ${ }^{16}$ separation of submicrometer sized microspheres, ${ }^{17}$ size-dependent sorting of emulsion droplets, ${ }^{18}$ and recently we demonstrated PFF as a DNA analysis technique for the detection of single nucleotide polymorphisms. ${ }^{19}$

In this paper we demonstrate a method for enhancing separation in PFF. The method does not involve any added external forces, only a snakelike structure in the broadening segment of the PFF layout. The enhanced PFF (EPFF) device layout is shown in Fig. 1(b). The part of the broadening segment to the left of the snakelike structure is denoted as the sink segment and the part to the right is denoted as the enhancing segment. The distribution of hydraulic resistances ensures that only the fluid containing particles flow through the enhancing segment. As the fluid and particles move from the narrow into the wide part of the enhancing segment, the distance between the streamlines (trajectories of different sized particles) is amplified. By expanding distances prima-

${ }^{a)}$ Electronic mail: anders@mailaps.org. rily in the part of fluid that contains particles and not in the entire fluid as in PFF, the enlargement and thereby separation of particles can be increased.

Yamada et al. suggested a simple model to describe PFF. The model is derived from the linear Stokes equation and conservation of mass relating the distance from the center of a particle to the channel wall in the broadening segment $\left(y_{\mathrm{PFF}}\right)$ and the radius of the particle $\left(r_{p}\right)$, [see Eq. (1)].

$$
y_{\mathrm{PFF}}=\frac{w_{b}}{w_{p}} r_{p} .
$$

Here, $w_{b}$ is the width of broadening segment and $w_{p}$ is the width of the pinch segment.

In this one-dimensional model it is assumed that the flow is laminar, that the particle is completely pinched before it enters the broadening segment, and that there is a large width to height ratio in the pinch and broadening segment $\left(w_{p} \gg h\right.$ and $w_{b} \gg h$ ). Furthermore, all effects from the particles on the fluid and particle flow, such as particle-fluid, particle-wall and particle-particle interactions are neglected.

Using the same assumptions as those behind Eq. (1), a simple model can be made for the EPFF device, relating the

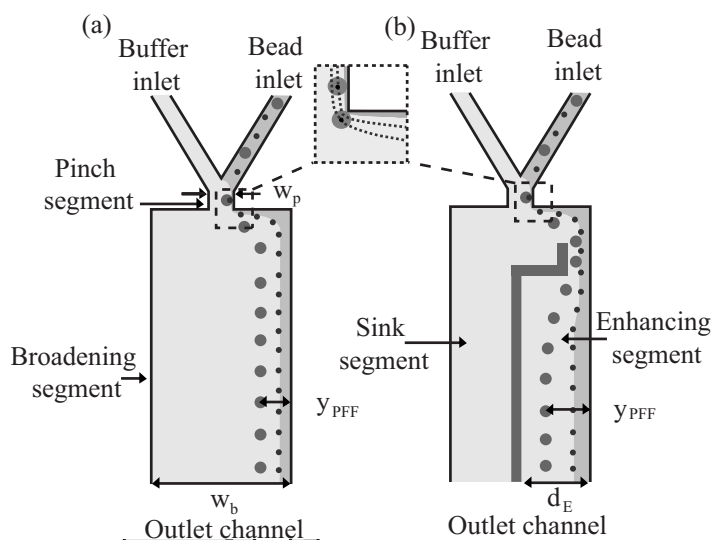

FIG. 1. The principle of (a) PFF and (b) EPFF. Suspended particles are aligned (pinched) against a wall in the pinch segment and separated as they move into the broadening segment due to expansion of the laminar flow sheet. In EPFF the separation is amplified by expanding the part of flow sheet that contains particles. The inset illustrates the so-called corner effect. 


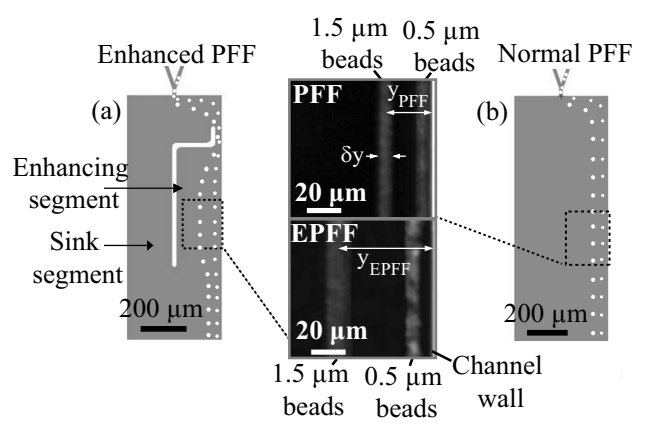

FIG. 2. Separation of polystyrene microspheres with radii of 0.5 and $1.5 \mu \mathrm{m}$ in (a) a PFF device and (b) an EPFF device. Each bead size is displaced further from the wall and the separation is amplified in EPFF with regard to the PFF device.

distance from the center of mass of a particle to the channel wall in the broadening segment $\left(y_{\mathrm{EPFF}}\right)$ and the radius of the particle $\left(r_{p}\right)$ [see Eq. (2)],

$$
y_{\mathrm{EPFF}}=\left(\frac{R_{E}}{R_{s}}+1\right) \frac{d_{E}}{w_{p}} r_{p}
$$

Here $d_{E}$ is the width of the wide segment in the enhancing segment [see Fig. 1(b)] $R_{E}$ is the total hydraulic resistance of the enhancing segment, and $R_{S}$ is the total hydraulic resistance of the sink segment. The hydraulic resistances of channels having rectangular cross sections are calculated as described in Ref. 20.

Separation in PFF and in EPFF can be compared by the separation enhancement factor (SEF) [see Eq. (3)],

$$
\mathrm{SEF}=\frac{y_{\mathrm{EPFF}}}{y_{\mathrm{PFF}}}=\left(\frac{R_{E}}{R_{s}}+1\right) \frac{d_{E}}{w_{b}} .
$$

As seen from Eq. (3), we expect the SEF only to depend on geometrical values and to be independent of the particle size.

As observed and discussed by Jain and Posner ${ }^{21}$ the linear model for PFF [Eq. (1)] overestimates the distance between particles and the wall in the broadening segment. Our data (see below) are consistent with the observations of Jain and Posner. We therefore present a semi-three dimensional (3D) numerical calculation of the flow in the PFF and EPFF device. However, still assuming that the particles do not perturb the flow. The only particle effect that is taken into account is a corner effect where the pinch segment ends out in the broadening segment, as illustrated in the inset of Fig. 1. At this point the streamlines in the fluid is forced closer to the wall compared to their position in the pinch segment. Particles will therefore be forced to follow streamlines further into the fluid, and thereby end longer from the wall in the broadening segment than expected if you only consider the particle position in the pinch segment. COMSOL MULTIPHYSICS $^{22}$ is used to perform the numerical calculation. In the calculation we have added no-slip boundary conditions at all channel walls and the flow rates in the two inlet channels are set to the same value as used in the measurements, 5 and $600 \mu \mathrm{l} / \mathrm{h}$ in the bead and buffer inlet channels, respectively. Furthermore, a volume force, $\bar{F}=-a * \bar{v}$, was introduced in the opposite direction of the velocity field, $\bar{v}$, to compensate for the hydraulic resistance from the bottom and top surface of the channel. $a=12 \eta / h, \eta$ is the viscosity of the buffer and $h$ is the height of the channels. The finite
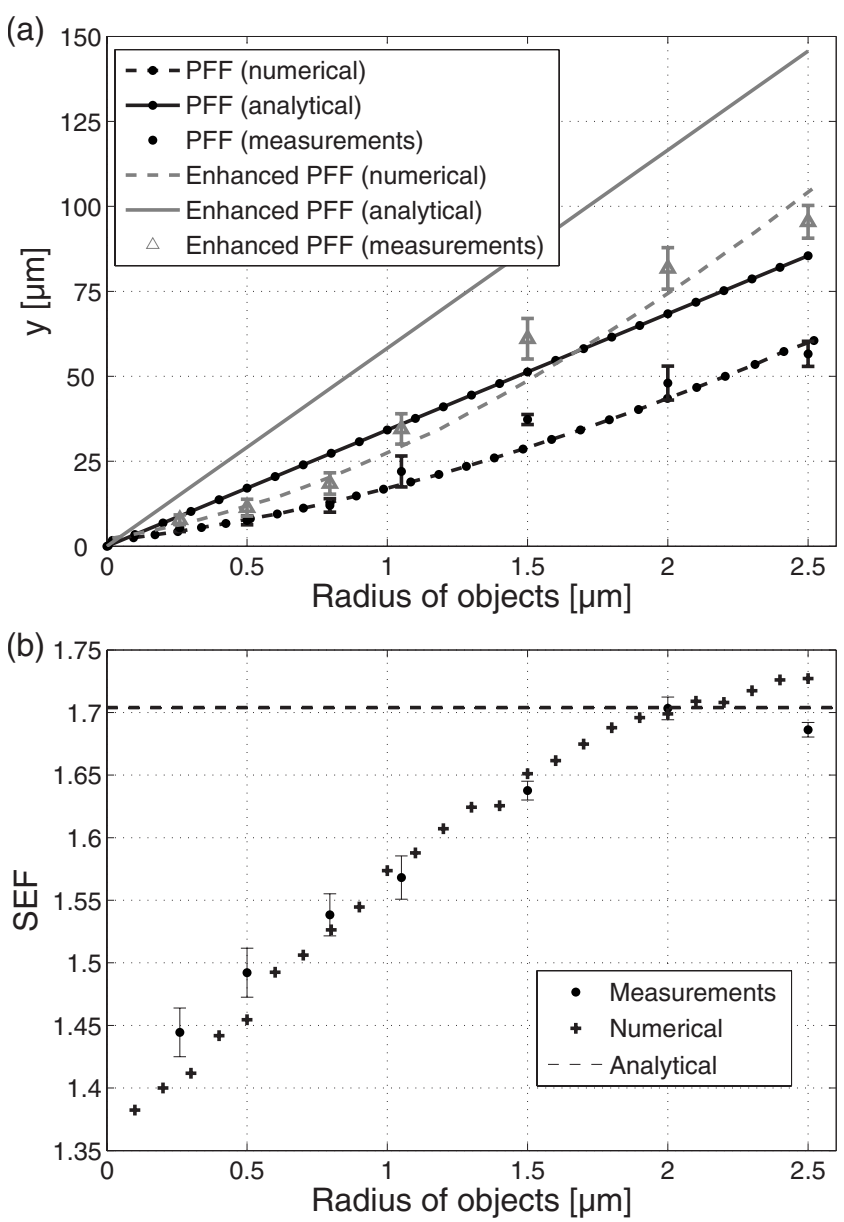

FIG. 3. (a) Distance between the bead position in the broadening segment and the channel wall $y_{\mathrm{PFF}}$ and $y_{\mathrm{EPFF}}$ in the PFF and EPFF device, respectively, as a function of the particle radius. Each measurement data point represents $>300$ beads, and the error bars represents the standard deviation of the measurements. (b) The SEF as a function of the particle radius.

height of the device is thereby taken into the model, which is therefore denoted as semi-3D.

The numerically calculated $y_{\mathrm{PFF}}, y_{\mathrm{EPFF}}$ and SEF are plotted as a function of the particle radius in Fig. 3 and do not predict $y_{\mathrm{PFF}}$ and $y_{\mathrm{EPFF}}$ to be linearly depending on the radius or SEF to be constant as predicted from the analytical models, see Eqs. (1)-(3), respectively.

Devices based on PFF and EPFF were fabricated in a cyclo-olefin copolymer (COC) called mr-I T85-20XP from Micro resist technology ${ }^{23}$ using nanoimprint lithography (NIL) as described in Ref. 19. The microchannels in both types of devices are made $12.5 \mu \mathrm{m}$ deep, and the width ranges from $12 \mu \mathrm{m}$ (the pinch segment) to $400 \mu \mathrm{m}$ (the broadening segment). The only thing that differs in the two devices is the snakelike structure. The narrow part of the enhancement segment is $25 \mu \mathrm{m}$ wide and $90 \mu \mathrm{m}$ long and the wide part is $190 \mu \mathrm{m}$ wide and $510 \mu \mathrm{m}$ long. The EPFF device is constructed so that microspheres with a radius of up to $4.5 \mu \mathrm{m}$ will enter the enhancing segment.

Separation measurements were conducted in the PFF and EPFF with seven different sizes of polystyrene microspheres ranging in radius between 0.25 and $2.5 \mu \mathrm{m}$. The microspheres were suspended in a buffer liquid (milli-Q water with $0.1 \mathrm{wt} \%$ SDS) and identical buffer was used as the pinching liquid. The flow rates in the two inlet channels were set to 5 and $600 \mu \mathrm{l} / \mathrm{h}$ in the bead and buffer inlet, respec- 
tively. All measurements were carried out with a bead concentration of $0.05 \mathrm{vol} \%$. Separation of 0.5 and $1.5 \mu \mathrm{m} \mathrm{mi-}$ crospheres in the PFF and EPFF device is shown in Fig. 2.

It is evident in Fig. 2 that separation of 0.5 and $1.5 \mu \mathrm{m}$ spheres is enhanced in the EPFF [Fig. 2(a)] compared to that in the PFF device [Fig. 2(b)]. Since the enhancing segment amplify the distance between the streamlines, the sphere stream width $(\delta y)$, as indicated in Fig. 2, will increase with increasing separation. The measured position of the beads in the broadening segment, $y_{\mathrm{PFF}}$ and $y_{\mathrm{EPFF}}$, of the seven bead sizes is plotted together with the analytical and numerical calculations in Fig. 3(a). The SEF of each bead size is plotted in Fig. 3(b) together with the analytical and numerical calculations.

As seen in Fig. 3(a) the numerical calculation fits much better with the measurements compared to the analytical predictions from Eqs. (1) and (2). The analytical calculations overestimate the measured values of $y_{\mathrm{PFF}}$ and $y_{\mathrm{EPFF}}$ up to $60 \%$, consistent with the reported results in Ref. 21. The numerical calculation fits very well with the results from the smallest beads but begin to deviate more as the bead size increases, with a maximum deviation of $23 \%$. This discrepancy is most likely due to particles perturbing the fluid flow. However, the presented numerical calculation represents a quantitatively good description of the results. The measured SEFs, plotted in Fig. 3(b), fits well with the numerical values, whereas the constant analytical value predicts an upper limit of the SEF. The measurements revealed an enhancement in separation that increases with the radius of the particles, saturating at approximately $70 \%$.

In conclusion it has been shown that the separation in PFF can be enhanced by a simple geometric modification of the device layout. This amplification does not rely on any external forces-which usually add complexity to the fabrication process. The fabricated enhanced PFF devices is therefore suited for cheap and high volume production methods such as NIL. In addition a semi-3D numerical model was developed to describe separation in the system. The SEFs were found to be dependent on particle size and range up to $70 \%$, in good accordance with the developed model.

The authors acknowledge economical support from the Toubro foundation and Fredrik Persson for theoretical discussion and proofreading.

${ }^{1}$ D. Janasek, J. Franzke, and A. Manz, Nature (London) 442, 374 (2006).

${ }^{2}$ A. Manz, J. C. Fettinger, E. Verpoorte, H. Lüdi, H. M. Widmer, and D. J. Harrison, Trends Analyt. Chem. 10, 144 (1991)

${ }^{3}$ J. C. Giddings, Science 260, 1456 (1993).

${ }^{4}$ S. Oh, D. Kang, S.-M. Ahn, R. J. Simpson, B.-H. Lee, and M. H. Moon, J. Sep. Sci. 30, 1082 (2007).

${ }^{5}$ S. Kim, D. Y. Kang, S. Lee, W.-S. Kim, J.-T. Lee, H. S. Cho, and S. H. Kim, Journal of Liquid Chromatography and Related Technologies 30, 2533 (2007).

${ }^{6}$ X. Huang, R. S. Mclean, and M. Zheng, Anal. Chem. 77, 6225 (2005).

${ }^{7}$ M. T. Blom, E. Chmela, R. E. Oosterbroek, R. Tijssen, and A. van den Berg, Anal. Chem. 75, 6761 (2005).

${ }^{8}$ Y. Okamoto, F. Kitagawa, and K. Otsuka, Electrophoresis 27, 1031 (2006).

${ }^{9}$ H. Ghourchian and H. Elyasvandi, Med. Eng. Phys. 27, 723 (2005).

${ }^{10}$ M. P. MacDonald, G. C. Spalding, and K. Dholakia, Nature (London) 426, 421 (2003).

${ }^{11}$ L. R. Huang, E. C. Cox, R. H. Austin, and J. C. Sturm, Science 304, 987 (2004).

${ }^{12}$ I. Doh and Y.-H. Cho, Sens. Actuators, A 121, 59 (2005).

${ }^{13}$ N. Pamme and C. Wilhelm, Lab Chip 6, 974 (2006).

${ }^{14}$ N. Pamme, Lab Chip 7, 1644 (2007).

${ }^{15}$ M. Yamada, M. Nakashima, and M. Seki, Anal. Chem. 76, 5465 (2004).

${ }^{16}$ J. Takagi, M. Yamada, M. Yasuda, and M. Seki, Lab Chip 5, 778 (2005).

${ }^{17}$ Y. Sai, M. Yamada, M. Yasuda, and M. Seki, J. Chromatogr., A 1127, 214 (2006).

${ }^{18}$ H. Maenaka, M. Yamada, M. Yasuda, and M. Seki, Langmuir 24, 4405 (2008).

${ }^{19}$ A. V. Larsen, L. Poulsen, H. Birgens, M. Dufva, and A. Kristensen, Lab Chip 8, 818 (2008).

${ }^{20} \mathrm{H}$. Bruus, Theoretical Microfluidics (Oxford University Press, Oxford, 2008).

${ }^{21}$ A. Jain and J. D. Posner, Anal. Chem. 80, 1641 (2008).

${ }^{22}$ See http://www.comsol.com/.

${ }^{23} \mathrm{GmbH}$, Berlin, Germany (www.microresist.de). 